\title{
6 Umweltinformationssysteme und -management
}

\subsection{Umweltdaten und Informationsmanagement}

Gregor Laaha ${ }^{a}$, Johannes Schmidt ${ }^{b}$ und Sebastian Wehrle ${ }^{b}$

a Institut für Statistik, Department für Raum, Landschaft und Infrastruktur (RALI)

${ }^{b}$ Institut für Nachhaltige Wirtschaftsentwicklung, Department für Wirtschafts- und Sozialwissenschaften (WiSo)

gregor.laaha@boku.ac.at, johannes.schmidt@boku.ac.at, sebastian.wehrle@boku.ac.at

\subsubsection{Einleitung}

In diesem Beitrag werden die Bereiche Umweltdatenmanagement und Umweltstatistik vorgestellt und ihre Anwendung in der Umweltökonomie beispielhaft gezeigt. Dafür sind Kenntnisse und Fertigkeiten zu Management, Modellierung und Bewertung von Umweltdaten mit Raum- und Zeitbezug wichtig. Im Bachelorstudium UBRM werden dazu Grundlagen vermittelt, die im Masterstudium in einem eigenen Fachbereich vertieft werden können. Die vermittelten Fertigkeiten beinhalten Datenhaltung und management, Visualisierung und Analyse mittels Geoinformationssystemen (GIS), Grundlagen des Modellierens und Simulierens, statistische Modellierung für Umweltdaten und deren Extremwerte sowie Methoden zur Bewertung von Umweltdaten und zur umweltökonomischen Entscheidungsunterstützung. Aufgrund seiner Anwendungsbreite liefert der Fachbereich einen Beitrag zu einer Reihe von SDGs, insbesondere für SDG 13 (Maßnahmen zum Klimaschutz), SDG 7 (bezahlbare und saubere Energie), SDG 15 (Leben an Land) und SDG 14 (Leben unter Wasser).

\subsubsection{Umweltdaten}

Umweltstatistische Untersuchungen sind größere Aufgabenstellungen, die eine Reihe von Arbeitsschritten erfordern. Umweltdaten müssen zunächst gemessen oder erhoben, gesammelt und gespeichert werden. Darauf folgt die Aufbereitung der Daten und die Ableitung spezifischer Informationen, die dann als Ausgangspunkt für statistische Analysen und ökonomische Bewertungen dienen. Meist liegen diese Arbeitsschritte nicht in einer Hand, was zu einer Reihe von Problemen bei den Untersuchungen führen kann (Stoyan und Jansen 2013).

Der Umgang mit Umweltdaten ist eine komplexe Aufgabenstellung, da sehr heterogene Daten verschiedener Herkunft und Erfassungsart analysiert werden. Die Messungen 
sind in unterschiedlichem Maße mit Unsicherheiten (z.B. Messfehlern) behaftet. Oft werden auch nicht die Messwerte selbst, sondern daraus abgeleitete Größen analysiert, die zusätzliche Fehlerkomponenten beinhalten können. Ein Beispiel dafür ist die Bestimmung von Abflussmengen in der Hydrologie. Tatsächlich gemessen werden dabei Wasserstände, die über eine mathematische Beziehung zwischen Wasserstand und Abfluss (die sogenannte Pegelschlüsselkurve) erst in Abflusswerte umgerechnet werden müssen. Die zeitliche Veränderlichkeit dieser Beziehung führt zu zusätzlichen Fehlern, die den eigentlichen Messfehler um ein Vielfaches übertreffen können.

Eine weitere Besonderheit von Umweltdaten ist ihre zeitliche Variabilität, die dazu führt, dass Messungen nicht wiederholbar sind. Ein Ausfall von Messgeräten kann gerade bei Extremereignissen auftreten und führt zu Fehlwerten (Missing Values), die die Analysen substanziell beeinträchtigen können. Technischer Fortschritt und Lernprozesse verändern die Messmethodik, die Genauigkeit und die zeitliche Auflösung der Messungen, was die Vergleichbarkeit von Messreihen über einen längeren Zeitraum erschwert. In Abhängigkeit von der untersuchten Fragestellung sind unterschiedliche zeitliche Auflösungen der Daten (beispielsweise Stunden-, Tages-, oder Monatswerte bzw. zeitlich hochauflösende Daten) erforderlich, die bei der Messung und Modellierung berücksichtigt werden müssen. Dazu wird oft eine Glättung oder Mittelung der Daten vorgenommen, um zufällige Schwankungen zu eliminieren und so stabilere oder aussagekräftigere Werte zu erhalten.

Die großen Datenmengen umweltstatistischer Studien führen zu weiteren Problemen. Beim Ablegen der Daten erfolgt oft eine Kodierung. Für ihre Interpretation sind zusätzliche Informationen (sogenannte Metadaten) erforderlich, die oft nicht in ausreichendem Maße erfasst werden und dann zu Fehlinterpretationen führen können. Hinter den Daten stehen weitere Informationen, die oft nur den mit der Messung betrauten Personen bekannt sind. Eine Einbeziehung solcher Informationen als „Soft Data" ist lohnend, aber aufgrund der komplexen Datenstruktur schwierig und bedarf besonderer Konzepte.

Nicht zuletzt besteht an Umweltstudien ein großes öffentliches Interesse, das aber die Verfügbarkeit von Daten oder die Publizierbarkeit von Studien beeinträchtigen kann. So besteht laut Umweltinformationsgesetz (UIG 1993) zwar ein allgemeines Recht auf einen einfachen Zugang zu Umweltinformation und Daten, das in der Praxis aber oft durch Geheimhaltungsinteressen (wie z.B. öffentliche Sicherheit oder Geschäfts- und Betriebsgeheimnisse) eingeschränkt wird. Viele Menschen interessieren sich für Umweltprobleme und verbinden mit umweltstatistischen Untersuchungen Hoffnungen oder Befürchtungen, was den sachlichen Umgang mit Informationen erschwert. So können die Auswertungsergebnisse zu beachtlichen Konse- 
quenzen führen, wenn negative Aussagen rufschädigend wirken oder das Risiko von Missverständnissen besteht.

Vor Beginn einer Studie sollte daher einer Reihe von Informationen eingeholt werden:

- Herkunft der Daten,

- Motivation der Datenerhebung,

- Darstellung, Genauigkeit und Zuverlässigkeit der Daten,

- Aufwand und Nutzen der Messungen.

Das hilft, die enthaltene Information und die Unsicherheiten besser einzuschätzen und den Umgang mit den Daten festzulegen. Umweltdaten können durch terrestrische (erdgebundene) Messungen bzw. Messnetze sowie Fernerkundungsverfahren gewonnen werden. In diesem Abschnitt wird auf Aspekte von terrestrischen Messungen sowie die statistische Analyse und ökonomische Bewertung von Umweltdaten eingegangen. Für eine spezifische Diskussion von Fernerkundungsdaten und Geoinformationen siehe Beitrag 6.2.

\subsubsection{Umweltstatistik}

\subsubsection{Charakteristik}

Im Rahmen einer umweltstatistischen Studie sollen Schlüsse über den Zustand der Umwelt gezogen werden. Dabei stehen häufig folgende Fragestellungen im Mittelpunkt:

- Was ist der aktuelle Zustand der Umwelt (Monitoring)?

- Hat sich der Zustand in den letzten Jahren oder Jahrzehnten verändert (Homogenität, Trend)?

- Wie wird sich der Zustand in naher Zukunft (Kurzzeitprognose), mittelfristig (saisonale Vorhersage) oder über einen längeren Zeithorizont (Langzeitprognose, Klimaprojektion) entwickeln?

- Wie groß ist das Risiko, dass eine Gefahrensituation, wie z.B. ein Hochwasserereignis oder eine Grenzwertüberschreitung eines Luftschadstoffs eintritt (Extremwertstatistik)?

- Gibt es Zusammenhänge zwischen dem Zustand der Umwelt und anderen Umwelt- bzw. Einflussgrößen (Korrelation)?

- Können diese Zusammenhänge für eine räumliche oder zeitliche Prognose (Schätzung) verwendet werden und falls ja, wie (statistisches Modell)?

Zur Beantwortung solcher Fragestellungen sind spezifische Umweltdaten erforderlich. Erschwerend ist, dass es sich bei Umweltphänomenen um komplexe räumlich- 


\section{Umweltinformationssysteme \& -management}

zeitliche Systeme handelt, die sich einer gesamtheitlichen Betrachtung weitgehend entziehen. Bei rein zeitlicher Betrachtung steht eine einzelne Messreihe $\boldsymbol{X}(t)$ im Mittelpunkt. Bei einer räumlichen Betrachtung werden Messungen $\boldsymbol{Z}$ an Orten mit den Lagekoordinaten $(x, y)$ (und manchmal der Seehöhe) berücksichtigt. Diese Daten sind mit Unsicherheiten wie Mess- und Auswertefehlern sowie einer gewissen Zufälligkeit (im Sinn von nicht erklärbaren Entstehungsprozessen) behaftet und werden daher als räumliche Zufallsvariable $\boldsymbol{Z}(x, y)$ bezeichnet.

Im Rahmen einer statistischen Studie will man Aussagen über die Daten als Ganzes treffen (kollektive Vorgehensweise). Hierbei ist auch der Umgang mit Unsicherheit entscheidend. Die Statistik als methodische Wissenschaft liefert eine Fülle an Verfahren zur Beschreibung und Analyse unsicherer Daten und Informationen. Sie bietet die Möglichkeit, eine systematische Verbindung zwischen Erfahrung (Empirie) und Theorie herzustellen. Bei der Umweltstatistik stehen angewandte, umweltwissenschaftliche Fragen im Mittelpunkt. Für ihre Beantwortung ist eine Auswahl spezieller Verfahren und Modelle erforderlich, die den Besonderheiten von Umweltdaten Rechnung trägt (Abbildung 6.1.1).

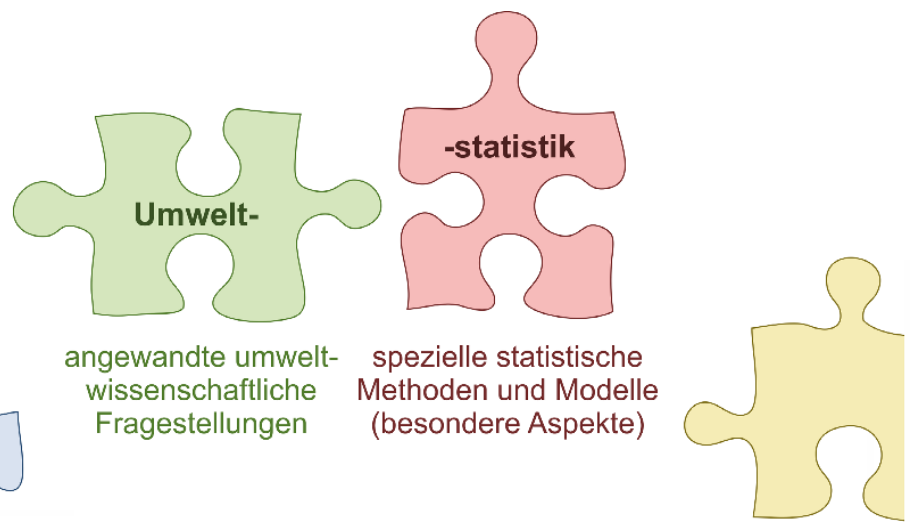

Abbildung 6.1.1: Umweltstatistik, spezielle Methoden für umweltspezifische Fragestellungen

\subsubsection{Systematik der Aufgabenstellungen}

Bei umweltstatistischen Studien können meist lokale und regionale Aufgabenstellungen unterschieden werden. Lokale Aufgaben beziehen sich auf einen Messpunkt ohne Berücksichtigung des regionalen Kontexts. Die Datengrundlage bildet meist eine Messreihe der interessierenden Größe. Eine typische Aufgabe liegt in der Angabe von langjährigen mittleren Kenngrößen (wie dem mittleren Jahresniederschlag oder der mittleren maximalen Lufttemperatur in einem Kalendermonat). Dazu werden statistische Kennzahlen wie arithmetischer Mittelwert, Minimum und Maximum, Standardabweichung 
oder Quantile (Werte mit bestimmter Auftretenswahrscheinlichkeit) herangezogen. Im Rahmen der Homogenitätsanalyse werden Messreihen auf zeitliche Veränderungen (Trends und Sprünge) untersucht, um etwa mögliche Auswirkungen des Klimawandels oder anthropogener Eingriffe zu analysieren. Bei der Extremwertstatistik stehen Extremereignisse und Naturgefahren wie Hochwässer oder Dürreereignisse im Blickpunkt. Soll der Zusammenhang zwischen verschiedenen Umweltgrößen untersucht werden, stehen Verfahren der Zeitreihenanalyse und Korrelationsanalysen zur Verfügung.

Bei den regionalen Aufgaben steht ein Messnetz von Pegelstellen oder Stationen im Mittelpunkt der Untersuchungen. Ziel ist es, aus dem räumlichen Muster der Messwerte räumlich geschlossene Aussagen abzuleiten. Um eine flächendeckende Kartierung der untersuchten Größe zu erstellen, werden Interpolationsverfahren herangezogen. In der Regel weisen Nachbarpunkte ähnlichere Werte auf als weit voneinander entfernte Punkte. Dieser Umstand wird als räumliche Korrelation bezeichnet und im Rahmen von geostatistischen Verfahren bei der Modellierung von Umweltphänomenen eingesetzt. Geostatistische Methoden ermöglichen eine Interpretation räumlicher $\mathrm{Zu}$ sammenhänge und können etwa im Rahmen von Umweltmonitoringprogrammen zur Schätzung von Flächenkontaminationen eingesetzt werden.

\subsubsection{Statistische Grundlagen}

Ausgangspunkt für die statistische Analyse ist ein beobachteter Datensatz über die interessierende Größe (Merkmal). Dabei ist es sinnvoll, nur einen repräsentativen Teil (Stichprobe) aller möglichen Daten (Grundgesamtheit) zu erheben. Die Wahl der statistischen Methode hängt wesentlich vom Skalenniveau des Merkmals ab. Prinzipiell unterscheidet man:

- kontinuierliche Merkmale, die auf einer metrischen Skala messbar sind, wie Temperatur in ${ }^{\circ} \mathrm{C}$, Regenintensität in $\mathrm{mm} / \mathrm{h}$, Abfluss in $\mathrm{m}^{3} / \mathrm{s}$, Pestizidbelastung in parts per million (ppm),

- diskrete Merkmale (Zählmerkmale und kategoriale Merkmale wie Geschlecht oder geologische Formation, Bodentyp, Landbedeckungsklasse, deren Ausprägungen in Form einer Kategorie angegeben werden).

Sind beobachtete Merkmale in einem Datensatz zusammengefasst worden, kann die Frage gestellt werden, wie häufig bestimmte Werte, Situationen oder allgemein Ereignisse vorkommen. Die absolute Häufigkeit $h_{a}$ erhält man durch Zählen aller Werte, die einem Ereignis entsprechen (z.B. Hochwässer mit einem Abfluss zwischen 110 und 120 m³/s). Sie ist von der Stichprobengröße (Anzahl $n$ ) abhängig. Dies erschwert eine Vergleichbarkeit von Stichproben. Es wird daher meistens die relative Häufigkeit $h_{r}$ verwendet, die sich durch Division der absoluten Häufigkeit durch die Stichprobenanzahl $n$ ergibt. 


\section{Umweltinformationssysteme \& -management}

Die Häufigkeitsverteilung einer Stichprobe kann als Histogramm (kontinuierliche Daten) oder Stabdiagramm (kategoriale Daten) dargestellt werden. Durch fortschreitendes Aufsummieren der Klassenhäufigkeiten erhält man das kumulierte Histogramm, das auch als Summenpolygon bezeichnet wird (Abbildung 6.1.2).
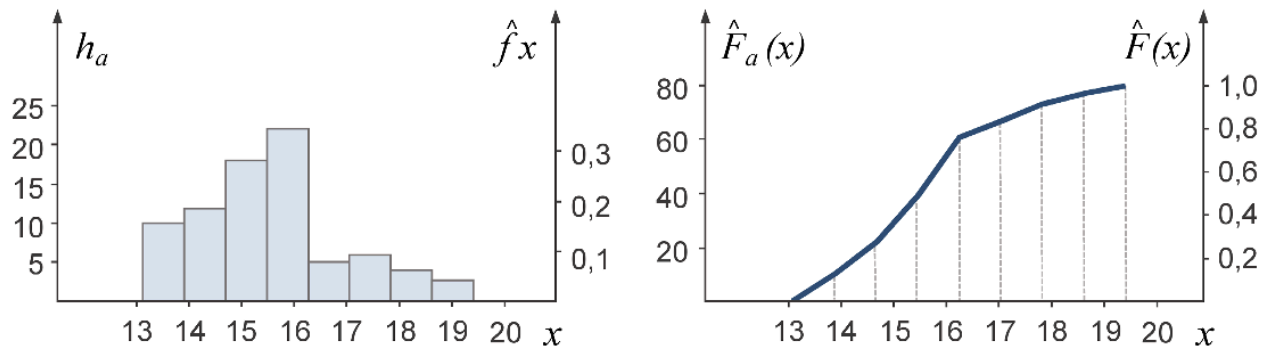

Abbildung 6.1.2: Nichtkumulierte und kumulierte Häufigkeitsverteilung der Stichprobe, Histogramm (links) und Summenpolygon (rechts)

Ziel der schließenden Statistik ist es, von der Stichprobe auf die Grundgesamtheit zu schließen (statistische Inferenz). Das Gesetz der großen Zahlen besagt, dass die relative Häufigkeitsverteilung mit wachsender Stichprobenanzahl in die Wahrscheinlichkeitsverteilung der Grundgesamtheit übergeht. Die Wahrscheinlichkeitsverteilung ist eine wichtige Kennlinie eines Merkmals, die eine komplette Beschreibung der Auftretenswahrscheinlichkeit von Ereignissen enthält. Auch sie wird in nichtkumulierter und kumulierter Form angegeben. Die nichtkumulierte Form heißt Wahrscheinlichkeits(dichte-)funktion $f(x)$ und entspricht dem Histogramm bzw. Stabdiagramm der Stichprobe. Sie gibt die relative Wahrscheinlichkeit von Merkmalswerten zueinander an und ermöglicht somit Aussagen der Form „Welche Körpergröße ist bei Männern wahrscheinlicher, $160 \mathrm{~cm}$ oder $180 \mathrm{~cm}$ ?". Die kumulierte Form heißt Verteilungsfunktion $F(x)$ und entspricht dem kumulierten Histogramm. Sie ermöglicht Aussagen über die Wahrscheinlichkeit von Wertebereichen oder „Ereignissen“, wie z.B. „Wie groß ist die Wahrscheinlichkeit einer Körpergröße $\leq 180 \mathrm{~cm}$ ?" (Abbildung 6.1.3).
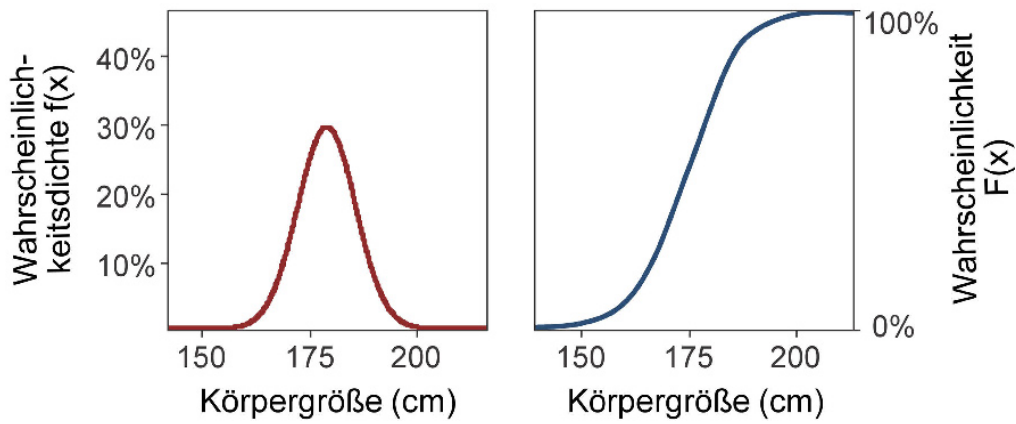

Abbildung 6.1.3: Wahrscheinlichkeitsverteilung: Dichtefunktion (links) und Verteilungsfunktion (rechts) 
In diesem Fallbeispiel soll die Anwendung der Wahrscheinlichkeitsrechnung im Rahmen einer extremwertstatistischen Studie gezeigt werden. Den Ausgangspunkt bilden Messreihen der täglichen Windspitzengeschwindigkeiten (nach der 12-teiligen Beaufortskala) in mehreren Messpunkten über Island (Stoyan und Jansen 2013). Anhand der Informationen an den Einzelpunkten wird ein räumlich aggregierter Sturmindex betrachtet, der die Anzahl der Stationen, an denen 9 Beaufort erreicht oder überschritten wurde, angibt. Die Tageswerte dieses Index liegen zwischen 0\% (kein Sturm) und 100\% (extremer Sturm über ganz Island). Sie bilden eine Zeitreihe, die in der Folge analysiert wird.

Mithilfe der Extremwertstatistik soll nun eine Einschätzung der jährlichen Auftretenswahrscheinlichkeit von Extremereignissen erfolgen. Dabei stehen typischerweise folgende Fragestellungen im Mittelpunkt:

- Welche Sturmstärke ist in einem durchschnittlichen Jahr zu erwarten?

- Wie hoch ist die Wahrscheinlichkeit, dass z.B. ein Sturmstärkeindex von 60 auftritt?

- Welche extreme Sturmstärke tritt im Durchschnitt nur alle 100 Jahre auf (100-jährliches Ereignis)?

Zur Beantwortung der Fragestellungen ist die Kenntnis der Wahrscheinlichkeitsverteilung der Extremereignisse erforderlich. Dazu wird zunächst eine Extremwertserie für den Beobachtungszeitraum 1912-1992 (81 Jahre) gebildet, die sich aus der maximalen Sturmstärke jedes Jahres zusammensetzt. Die Daten sind in Abbildung 6.1.4 als Histogramm dargestellt. Aufgrund der symmetrischen Form scheint eine Normalverteilung gut zu passen. Die Normalverteilung hat zwei Parameter, Mittelwert $\mu$ und Standardabweichung $\sigma$, die die Form der Verteilung bestimmen. Bei der Verteilungsanpassung werden sie durch den Stichprobenmittelwert $\bar{x}$ (hier ein Indexwert von 54,18 ) und die Stichprobenstandardabweichung $s$ (hier 13,70) geschätzt.

Die resultierende Verteilung ist in Abbildung 6.1.4 dargestellt. Die linke Graphik zeigt das Histogramm der Stichprobenwerte und die daran angepasste Wahrscheinlichkeitsdichtefunktion. Mit ihrer Hilfe kann die Frage nach der in einem durchschnittlichen Jahr zu erwartende Sturmstärke beantwortet werden. Diese entspricht dem Erwartungswert der Verteilung. Bei einer Normalverteilung entspricht der Erwartungswert dem Wert mit der höchsten Wahrscheinlichkeitsdichte. Durch Ablesen desjenigen Werts auf der $x$-Achse, dem der höchste Punkt der Dichtefunktion entspricht, erhält man einen Indexwert von etwas unter 60 (genau 54,2). Dies entspricht dem Stichprobenmittelwert.

Die rechte Graphik in Abbildung 6.1.4 zeigt das kumulierte Histogramm der Stichprobe und die daran angepasste Verteilungsfunktion. Mit ihrer Hilfe können Fragen der Auftretenswahrscheinlichkeit von Ereignissen beantwortet werden. Hierbei ist zu berücksichtigen, dass die Verteilungsfunktion die Unterschreitungswahrscheinlichkeit $P(x)$ angibt. Dies ist die Wahrscheinlichkeit für das Auftreten eines Sturmereignisses, welches kleiner als $x$ ist. Im Fall von Maxima wie extremen Stürmen, Hoch-
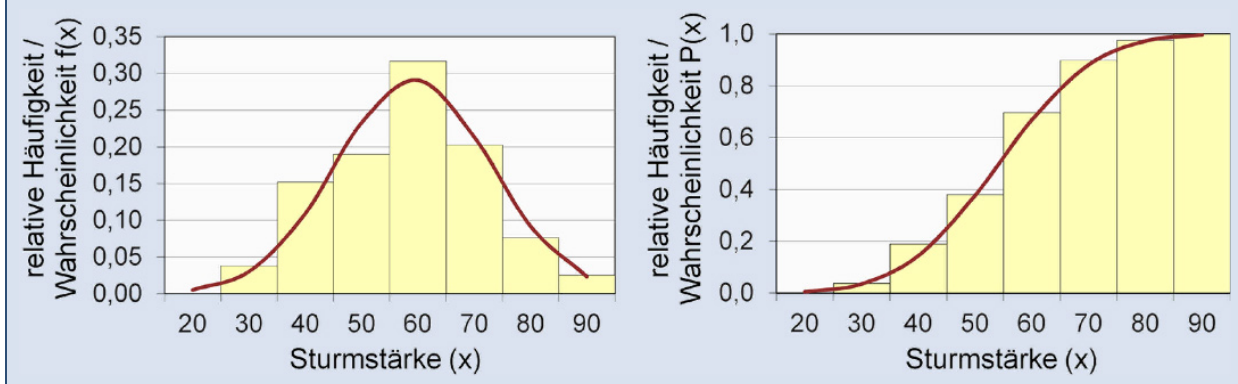

Abbildung 6.1.4: Empirische und theoretische Verteilung der maximalen Windstärken über Island, Links: Histogramm (gelb) und Wahrscheinlichkeitsdichtefunktion (rot), Rechts: Kumulatives Histogramm (gelb) und Verteilungsfunktion (rot) 
wässern etc. muss $P(x)$ in die Überschreitungswahrscheinlichkeit $P_{\ddot{u}}(x)=1-P(x)$ umgerechnet werden. In unserem Fall kann für den Sturmstärkeindexwert 60 eine Unterschreitungswahrscheinlichkeit von etwa 0,80 abgelesen werden. Daraus ergibt sich eine durchschnittliche jährliche Auftretenswahrscheinlichkeit von 0,20 (bzw. 20\%), d.h., in 20\% aller Jahre muss mit einer Sturmstärke > 60 gerechnet werden.

Die Frage nach einem 100-jährlichen Ereignis stellt eine Umkehraufgabe dar. Eine solche Aufgabe kann durch die Umkehrfunktion der Verteilungsfunktion, die sogenannte Quantilfunktion, beantwortet werden. Diese kann auf mathematischem Wege aus der Verteilungsfunktion abgeleitet werden. Hier wird eine graphische Lösung unter Verwendung der Verteilungsfunktion gezeigt. Ein 100-jährliches Sturmereignis ist als Windstärkeindex mit einer Überschreitungswahrscheinlichkeit von $p_{u ̈}=1 / 100=0,01$ definiert. Sie entspricht einer Unterschreitungswahrscheinlichkeit von $p=1-p_{u}=0,99$. Bei der graphischen Lösung geht man mit dem Wert von 0,99 auf der y-Achse in das Diagramm der Verteilungsfunktion und erhält das 100-jährliche Sturmereignis durch Ablesen des Werts auf der x-Achse. Die Umkehrfunktion wird also durch umgekehrtes Ablesen der Funktionswerte gebildet. Somit ergibt sich als 100-jährliches Ereignis eine Sturmstärke von etwa 85 (genau 86,1).

\subsubsection{Umweltökonomische Bewertung}

Umweltdaten und Umweltstatistik liefern wichtige Informationen, um umweltpolitische Entscheidungsprozesse zu unterstützen. Die Umweltökonomie stellt Werkzeuge und Modelle bereit, die die Bewertungen der Auswirkungen von Produktions- und Konsumverhalten auf die Umwelt ermöglichen. Dazu zählt die Bewertung

- der Wirkung von umweltpolitischen Maßnahmen auf die soziale Wohlfahrt und Umwelt,

- von externen Effekten bei Produktions- und Konsumaktivitäten,

- eines effizienten Managements von natürlichen Ressourcen und der Bereitstellung öffentlicher Güter (siehe dazu auch Beitrag 2.1).

Die umweltökonomischen Werkzeuge und Modelle beziehen in hohem Umfang Umweltdaten und umweltstatistische Methoden mit ein. Ein Beispiel dafür sind energieökonomische Modelle, die die Politik bei der Planung der Energiewende unterstützen. Sie berücksichtigen Klimadaten, um die Verfügbarkeit und Variabilität von erneuerbaren Energien besser abzuschätzen. Klima- und Wetterprognosen werden aber auch von betriebswirtschaftlichen Modellen im Energiesektor benötigt. Ein anderes Beispiel sind agrarökonomische Modelle, die beispielsweise verwendet werden können, um die Umweltauswirkungen von ökologischer und konventioneller Landwirtschaft auf die Biodiversität und auf das landwirtschaftliche Einkommen zu vergleichen. Hierfür sind umfangreiche Umweltdaten über den Zusammenhang zwischen agrarischer Produktion und Biodiversität notwendig.

Methodisch werden normative und positive Ansätze unterschieden. In normativen Ansätzen wird versucht, eine ökonomische Zielgröße effizient zu erreichen (z.B. die 
Reduktion von Treibhausgasemissionen (Schmidt et al. 2010)), während in positiven Modellen Umweltindikatoren und ökonomische Daten mit statistischen Methoden auf Zusammenhänge und Kausalitäten untersucht werden (z.B. zwischen Freihandelspolitik und globalen Treibhausgasemissionen (Islam et al. 2016)).

Natürliche Ressourcen sind sowohl Produktionsfaktor als auch Lieferant von Ökosystemdienstleistungen in unserem Wirtschaftssystem. Jedoch beeinträchtigen unsere Produktions- und Konsumaktivitäten die Umwelt. Umweltdaten sind in beiden Fällen relevant. So beeinflussen Klimavariablen die Produktivität in der Landwirtschaft genauso wie die Energieerzeugung mittels erneuerbarer Technologien. Auch für die Abschätzung der Folgen menschlichen Wirtschaftens auf die natürliche Umwelt sind Umweltdaten wichtig. Nur wenn Emissionen und daraus resultierende Schäden systematisch erhoben werden, können Rückschlüsse gezogen werden, welchen Einfluss verschiedene Formen des Wirtschaftens auf das System Erde haben. Ein klassisches Beispiel ist die Messung von Treibhausgasemissionen und die Bestimmung von globalen Durchschnittstemperaturen, die es erlauben, den Zusammenhang zwischen dem Wirtschaften, den damit verbundenen Emissionen und dem Klimawandel zu beschreiben. Während in den letzten Jahren in diesem Bereich große Fortschritte erzielt wurden und eine immer genauere Beschreibung des Beitrags unterschiedlicher Sektoren, Regionen und Länder an den globalen Treibhausgasemissionen möglich ist, bestehen in anderen Bereichen noch große Lücken. So ist z.B. die systematische, globale Erfassung von Biodiversitätsdaten in vielen Fällen noch nicht ausreichend fortgeschritten, um Wirkungszusammenhänge umfassend und zuverlässig abschätzen zu können.

Hohe Datenmengen sind v.a. auch dann notwendig, wenn Entscheidungen unter Unsicherheit getroffen werden müssen. Als vereinfachtes Beispiel kann das Schadensrisiko von Naturgefahren wie etwa Hochwasser, Dürre oder Sturmschäden dienen. Dieses Risiko entspricht der mit der Eintrittswahrscheinlichkeit gewichteten Schadenshöhe, wobei die Eintrittswahrscheinlichkeit von Extremereignissen mithilfe der Extremwertstatistik ermittelt werden kann. Ist z.B. das Schadensrisiko einer Überschwemmung größer als die Kosten eines Hochwasserschutzes, so ist eine Investition in eine Schutzmaßnahme sinnvoll. Um solche Risikobewertungen durchführen zu können, sind lange Zeitreihen von Umweltdaten notwendig, da Schadensereignisse meist nur selten auftreten. Umweltzustände verändern sich nicht nur räumlich, sondern auch über die Zeit (u.a. auch durch menschlichen Eingriff), was die Bewertung erschwert. So können beispielsweise bei der Verwendung sehr kurzer Zeitreihen von Hochwasserdaten langfristige, möglicherweise durch den Klimawandel ausgelöste Trends nicht erkannt werden. Die Entscheidung über Schutzmaßnahmen würde in diesem Fall auf falschen Erwartungen über die zukünftige Wahrscheinlichkeit solcher Ereignisse basieren. 
Eine Bewertung von öffentlichen Gütern und Umweltbeeinträchtigungen ist notwendig, um gesellschaftliche Entscheidungen treffen zu können (siehe Fallbeispiel 6.1.2). Dies ist in der Praxis mit hohen Unsicherheiten und ethischen Problemen behaftet, da notgedrungen die Bewertung von menschlichem Leben, von Biodiversität oder von zukünftiger Lebensqualität in solche Modelle einfließen muss.

\section{Fallbeispiel 6.1.2: Kosten und Emissionen der Energiewende in Deutschland und Österreich}

Das UBRM-Studium beschäftigt sich in vielen Fällen mit der Frage, wie wir unsere natürlichen Ressourcen nachhaltig bewirtschaften können. Dies ist eine typische normative ökonomische Fragestellung. Im diesem Beispiel untersuchen wir, mit welchen Kosten und welchen $\mathrm{CO}_{2}$-Emissionen die Energiewende in Deutschland verbunden ist. In ökonomischen Modellen gibt es verschiedene Möglichkeiten, die Auswirkungen wirtschaftlicher Aktivität auf die Umwelt miteinzubeziehen. Erstens kann die Optimierung von Wirtschaftssystemen die monetarisierten Umweltschäden und Verschmutzungsvermeidungskosten berücksichtigen. Als zweite Möglichkeit kann die Umweltverschmutzung mit maximalen Limits beschränkt werden, in unserem Beispiel also die maximal erlaubten Treibhausgasemissionen im Stromsystem. Wir setzen das Limit für Treibhausgasemissionen im Stromsystem auf jenen Wert für das Jahr 2030, der die Erreichung des „1,5 ${ }^{\circ} \mathrm{C}$-Ziels als realistisch erscheinen lässt (lineare Reduktionen der Treibhausgasemissionen im Stromsektor auf null von heute bis 2050).

Schließlich kann auch eine Mehrzieloptimierung vorgenommen werden: Anstatt eine einzelne, optimale Lösung zu bestimmen, wird die gewichtete Summe mehrerer alternativer Indikatoren, in diesem Fall Emissionen sowie Brennstoff- und Kapitalkosten des Systems, minimiert. Werden diese Gewichte verändert, ergeben sich unterschiedliche Lösungen, die den Trade-off zwischen den beiden Zielen anzeigen und so eine Entscheidungsgrundlage bieten.

In unserem Beispiel verwenden wir das Strommarktmodell medea (Wehrle und Schmidt 2018). Das Modell minimiert die Kosten der Bereitstellung von Strom in Deutschland und Österreich unter Berücksichtigung der Variabilität von Nachfrage und erneuerbarem Angebot - und das auf stündlicher Basis. Das Modell entscheidet sich für Investitionen in neue Technologien (z.B. Windkraft und Photovoltaik), wenn beispielsweise der Preis für $\mathrm{CO}_{2}$-Emissionen erhöht wird. Damit können Kosten und $\mathrm{CO}_{2}$-Emissionen von unterschiedlichen Varianten der Energiewende einfach berechnet werden. Medea verwendet umfangreiche Umweltdaten: Zur Modellierung des Ausbaus erneuerbarer Energien werden Klimadaten (Windgeschwindigkeiten und solare Strahlung) verwendet. Außerdem werden im Modell die $\mathrm{CO}_{2}$-Emissionen, die in der thermischen Stromproduktion (z.B. Gas- und Kohlekraftwerke) entstehen, gegen Umweltdaten, die in den nationalen Treibhausgasbilanzen veröffentlicht werden, validiert.

Abbildung 6.1.5 zeigt die Resultate von Szenarienrechnungen, die sich an den diskutierten Möglichkeiten einer Berücksichtigung von Umweltverschmutzung in ökonomischen Modellen orientieren. In Szenario 1 fixieren wir den Emissionspreis auf $160 € / t \mathrm{CO}_{2}$ (roter Punkt in der Graphik) - einem 2016 publizierten Wert für das Jahr $2030^{1}$ (Nordhaus 2017). In Szenario 2 limitieren wir die jährlichen $\mathrm{CO}_{2}$-Emissionen in der Stromproduktion auf 170 Mio. t $\mathrm{CO}_{2}$ (blauer Punkt in der Graphik) derzeit werden in Deutschland rund $280 \mathrm{Mio}$. $\mathrm{CCO}_{2}$ pro Jahr ausgestoßen. In Szenario 3 suchen wir optimale Kombinationen aus Emissionspreis und $\mathrm{CO}_{2}$-Emissionen durch Mehrzieloptimierung (schwarze Punkte durch Linie verbunden). Wird das Gewicht für die Emissionen erhöht, wird ein Punkt optimal, der weniger Emissionen, aber höhere Kapital- und Brennstoffkosten vorweist.

Abbildung 6.1.5 zeigt also deutlich den Trade-off zwischen niedrigeren $\mathrm{CO}_{2}$-Emissionen im Stromsystem und den damit verbundenen Kosten. 


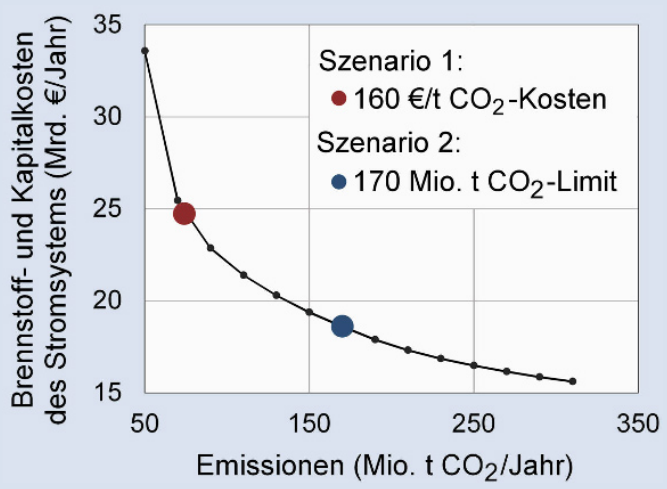

Abbildung 6.1.5: Ergebnisse der medea-Modellrechnungen zum Trade-off von Produktionskosten und $\mathrm{CO}_{2}$-Emissionen

Die Mehrzielanalyse gibt vielfältige Informationen über die Kosten der Dekarbonisierung des Energiesystems. So zeigt die Kurve beispielsweise, dass eine Reduktion von $70 \mathrm{Mio}$. $\mathrm{CO}_{2}$ auf $50 \mathrm{Mio}$. $\mathrm{CO}_{2}$ um ein Vielfaches teurer ist (8,13 Mrd. $€$ ) als eine Reduktion von $310 \mathrm{Mio}$. t $\mathrm{CO}_{2}$ auf $290 \mathrm{Mio}$. $\mathrm{C} \mathrm{CO}_{2}$ $(0,25$ Mrd. €). Eine vollständige Dekarbonisierung des Elektrizitätssystems ist also vermutlich kurzfristig nicht die billigste Option, um die gesamtwirtschaftlichen Treibhausgasemissionen zu reduzieren. Langfristig, bis 2050, sollten die $\mathrm{CO}_{2}$-Emissionen in der Stromproduktion allerdings auf 0 reduziert werden.

${ }^{1}$ Es gilt zu berücksichtigen, dass dieser Wert stark von den Annahmen abhängig ist und ethische Bewertungen inkludiert. Wir verwenden hier also einen Beispielwert, einer von vielen möglichen. Der Wert kann aber z.B. dafür verwendet werden, die eigenen, durch Treibhausgasemissionen verursachten Folgekosten abzuschätzen. Österreicherinnen und Österreicher verursachen im Jahr rund $15 \mathrm{t} \mathrm{CO}_{2}$ (konsumbasiert, im Jahr 2011 (Anderl et al. 2016)). Multipliziert mit dem $\mathrm{CO}_{2}$-Preis von $160 €$ ergibt das Kosten von $2.400 €$ pro Österreicherin oder pro Osterreicher.

\section{Literatur}

Anderl, M., Gössl, M., Kuschel, V., Haider, S., Gangl, M., Heller, C., Lampert, C., Moosmann, L., Pazdernik, K., Poupa, S., Purzner, M., Schieder, W., Schneider, J., Schodl, B., Stix, S., Stranner, G., Storch, A., Wiesenberger, H., Winter, R., Zechmeister, A. und Zethner, G. (2016): Klimaschutzbericht 2016. Wien: Umweltbundesamt.

Islam, M., Kanemoto, K., and Managi, S. (2016): Impact of trade openness and sector trade on embodied greenhouse gases emissions and air pollutants. Journal of Industrial Ecolology, 20, 3, 494-505. https://doi.org/10.1111/jiec.12455.

Nordhaus, W. D. (2017): Revisiting the social cost of carbon. Proceedings of the National Academy of Sciences of the United States of America, 114, 7, 1518-1523. https://doi.org/10.1073/pnas.1609244114.

Schmidt, J., Leduc, S., Dotzauer, E., Kindermann, G., and Schmid, E. (2010): Cost-effective CO2 emission reduction through heat, power and biofuel production from woody biomass: A spatially explicit comparison of conversion technologies. Applied Energy, 87, 7, 21282141. https://doi.org/10.1016/j.apenergy.2009.11.007.

Stoyan, H. und Jansen, U. (2013): Umweltstatistik: Statistische Verarbeitung und Analyse von Umweltdaten. Leipzig: Springer-Verlag.

UIG (1993): Bundesgesetz über den Zugang zu Informationen über die Umwelt (Umweltinformationsgesetz - UIG). i.d.F. Nov. 2018, zuletzt geändert mit BGBI. I Nr. 74/2018.

Wehrle, S. and Schmidt, J. (2018): District heating systems under high CO2 emission prices: the role of the pass-through from emission cost to electricity prices. https://arxiv.org/abs/1810.02109 [econ.GN]. 


\title{
6.2 Geoinformationssysteme und Fernerkundung
}

\author{
Anja Klisch, Thomas Bauer, Reinfried Mansberger und Clement Atzberger \\ Institut für Vermessung, Fernerkundung und Landinformation, \\ Department für Raum, Landschaft und Infrastruktur (RALI) \\ anja.klisch@boku.ac.at, t.bauer@boku.ac.at, mansberger@boku.ac.at, \\ clement.atzberger@boku.ac.at
}

\subsubsection{Einleitung}

Um den Verbrauch natürlicher Ressourcen sowie den Klimawandel und seine Auswirkungen zu verstehen, ist es wichtig, zu wissen, womit die Erdoberfläche heute bedeckt ist (man spricht von „Landbedeckung“, z.B. mit Vegetation, Bauwerken oder Gewässern) und wie sie genutzt wird.

Die Fernerkundung liefert diese Daten und ermöglicht damit die Erfassung des aktuellen Zustands der Erdoberfläche, z.B. über satellitengestützte Bilder und zugehörige Auswerteverfahren. Vergleicht man die aktuellen mit historischen Bilddaten, lässt sich auch ein Blick in die Vergangenheit werfen und die Veränderung der Landnutzung in Raum und Zeit untersuchen. Zur Speicherung, Analyse, Modellierung und Visualisierung solcher naturräumlichen Daten dienen Geoinformationssysteme (GIS).

In den folgenden Abschnitten wird ein Überblick darüber gegeben, welche Daten für das UBRM relevant sind und wie sie gewonnen und analysiert werden. Ebenso wird die Aufgabe von Koordinatensystemen beschrieben, die für die Verknüpfung von Daten von Bedeutung sind.

\subsubsection{Geodaten}

Daten, die einen räumlichen Bezug zu Objekten auf der Erde haben, werden als Geodaten bezeichnet. Der räumliche Bezug kann über geographische Namen, Adressen, Grundstücksnummern oder auch durch Koordinaten hergestellt werden. Durch Verknüpfung von Geodaten (Modellierungen, Simulationen) können neue Informationen gewonnen werden.

Der Fachbereich Vermessung, Fernerkundung und Landinformation umfasst die in Abbildung 6.2.1 dargestellten Wissenschaftsdisziplinen. Hier werden raumzeitliche Informationen geometrisch und thematisch erfasst, mithilfe von GIS gespeichert und analysiert sowie in Karten und Plänen dargestellt.

Welches Gebiet die Daten abdecken sollen (einen Betrieb, eine Region, eine Nation, einen Kontinent, den ganzen Globus) und wie detailliert sie räumlich, zeitlich und thematisch erfasst werden sollen, hängt vom jeweiligen Anwendungsgebiet ab. 


\section{Fernerkundung}

Gesamtheit der Verfahren, die es gestatten, aus großer Entfernung berührungsfrei Information über Art und Eigenschaften von Objekten zu erhalten
Geoinformations-

systeme

Systeme zur Erfassung, Speicherung, Analyse und Darstellung von raumbezogenen Daten
Photogrammetrie

manuelle und automatische Verfahren zur geometrischen Vermessung von Objekten aus photographischen Bildern

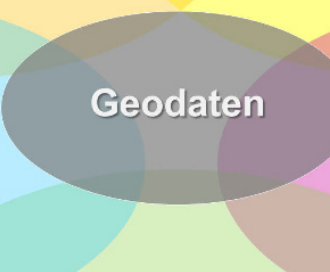

\section{Vermessung Verfahren der geometrischen
rermessung größerer oder kleinere Teile der Erdoberfläche und ihrer Darstellung in Plänen und Karten}

\section{Abbildung 6.2.1: Einordnung der für die Erfassung und Analyse von Geodaten relevanten Wissenschaftsdisziplinen}

Eine Möglichkeit, Geodaten zu erfassen, sind terrestrische Erhebungen (Vermessungsarbeiten, Taxation und andere messkundliche Aufnahmen). Diese Aufnahmeverfahren liefern differenzierte und genaue Daten. Sie sind jedoch sehr aufwendig zu gewinnen und liegen daher oft nicht aktuell und nicht flächendeckend vor. Neben der direkten Aufnahme vor Ort können Geodaten auch indirekt aus Luft- oder Satellitenbildern abgeleitet werden. Und schließlich werden mittlerweile sehr viele Geodaten von öffentlichen und privaten Institutionen auf Geoportalen angeboten, auf die gratis oder kostenpflichtig zugegriffen werden kann. Ein Beispiel für Geodaten aus öffentlichen Institutionen ist basemap.at (Stadt Wien et al. 2016) (siehe Abbildung 6.2.2), eine Grundkarte von Österreich, basierend auf den Geodaten von den Ländern und deren Partnern.

Aus Luft- und Satellitenbildern sowie Aufnahmen von „Airborne Laserscannern“ (ALS) können Geodaten berührungsfrei und großflächig gewonnen werden. Stehen geometrische Eigenschaften im Vordergrund, sprechen wir von Photogrammetrie - geht es dagegen um die thematische Erfassung von Daten, ist von Fernerkundung die Rede. Raumbezogene Daten werden zumeist in einem GIS verarbeitet, in digitaler Form gespeichert und für konkrete Anwendungen eingesetzt. Ältere Daten liegen oft noch als Ausdruck auf Papier oder Folie vor (z.B. konventionelle Karten; historische Orthophotos, also verzerrungsfreie Darstellungen der Erdoberfläche).

Im Zusammenhang mit Geodaten spielen auch „Metadaten“ eine große Rolle. Metadaten sind „Daten über Daten“, sie werden zur Beschreibung des Inhalts, der Quali- 


\section{Umweltinformationssysteme \& -management}

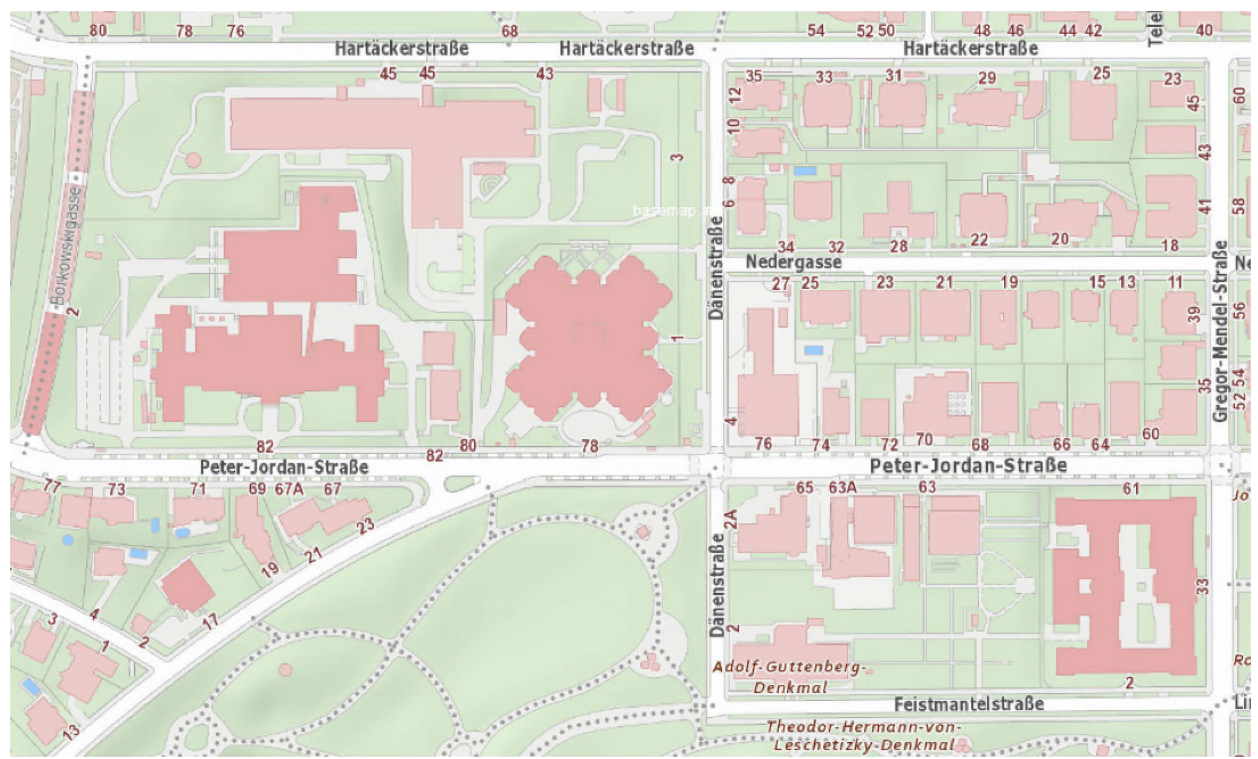

Abbildung 6.2.2: Ausschnitt aus basemap.at (BOKU - Türkenschanze) (Stadt Wien et al. 2016)

tät, der Nutzbarkeit, der Verfügbarkeit und anderer Eigenschaften von Daten verwendet. Die zunehmende Verfügbarkeit von Geodaten und die stetig steigende Zahl an Nutzerinnen und Nutzern, die nach geeigneten Geodaten suchen, erhöht den Bedarf nach Aussagen über die Datenbestände.

\subsubsection{Koordinatensysteme}

Der unmittelbare Gegenstand der Erfassung von Geodaten ist die sichtbare (physische) Erdoberfläche. Diese ist unregelmäßig gestaltet und weder mathematisch noch physikalisch exakt beschreibbar. Um aber dennoch räumliche Objekte weltweit eindeutig verorten zu können, werden als Näherung sogenannte Bezugsflächen verwendet.

Das Geoid ist eine physikalische Bezugsfläche und kommt der wahren Erdfigur am nächsten. Es leitet sich aus dem Erdschwerefeld ab. Entlang der Erdoberfläche können Flächen gleichen Schwerepotenzials definiert werden. Das Geoid entspricht derjenigen Fläche gleichen Schwerepotenzials, welche mit der ruhenden mittleren Meeresoberfläche zusammenfällt. Da die Masse an der Erdoberfläche und im Erdmantel unterschiedlich verteilt ist, entsteht die unregelmäßige Form des Geoids (siehe Abbildung 6.2.3a). Es wird als Bezugsfläche für Höhenangaben verwendet, wie z.B. Höhen über dem Meeresniveau bzw. in Österreich über „Adria Null“.

Möchte man lediglich die Lage oder Geometrie von Objekten auf der Erde beschreiben, werden sogenannte regelmäßige, mathematische Bezugsflächen verwendet. Für 


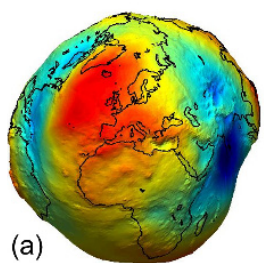

(a)

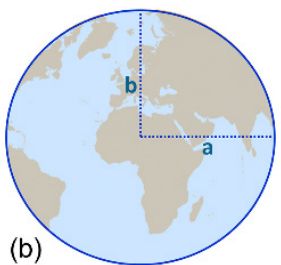

(c)
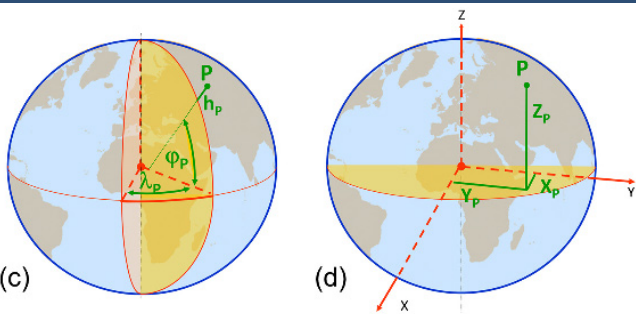

Abbildung 6.2.3: Bezugsflächen der Erde: (a) das Geoid als physikalische Bezugsfläche (ESA 2018a), (b) das Ellipsoid als mathematische Bezugsfläche, (c) ellipsoidische Koordinaten zur Verortung eines Punktes $P$ auf einem Ellipsoid und (d) kartesische Koordinaten zur Verortung eines Punktes $\boldsymbol{P}$ auf einem Ellipsoid (administrative Daten: GADM 2018)

kleinmaßstäbige Darstellungen großer Gebiete wie Kontinente in Atlanten kann eine Kugel als Bezugsfläche herangezogen werden.

Für die detailliertere Beschreibung von Objekten auf der Erdoberfläche muss zusätzlich die Erdabplattung berücksichtigt werden. Dies wird mithilfe von Ellipsoiden erreicht, die entweder weltweit oder für bestimmte Bereiche, wie das Staatsgebiet Österreichs, an die Erdoberfläche angepasst werden. Zur genauen Definition muss zum einen die Größe in Form der beiden Halbachsen bekannt sein (Abbildung 6.2.3b), zum anderen muss die Lagerung des Ellipsoids bezüglich der Erde genau definiert sein. Zur Verortung von Objekten auf einem Ellipsoid werden entweder ellipsoidische Koordinaten (Abbildung 6.2.3c) oder rechtwinkelige Koordinaten (Abbildung 6.2.3d) verwendet.

Aber wie gelangt man vom dreidimensionalen Erdkörper zu einer ebenen Karte oder einem Plan? Gleich vorab: Dieses Problem ist zwar unlösbar, es wurden aber verschiedene Kartenprojektionen entwickelt, um das Problem näherungsweise zu lösen. Aufgrund der Krümmung der Erdoberfläche und der zuvor beschriebenen mathematischen Bezugsflächen kann eine Abbildung in die Ebene nie verzerrungsfrei erfolgen. Verzerrungen können hinsichtlich Winkel, Strecken oder Flächen auftreten. Es wurde eine Vielzahl von Projektionen (Abbildungsvorschriften) entwickelt. Eine wichtige Rolle spielen hierbei Projektionsflächen. Dafür sind geometrische Formen geeignet, die bereits eben sind oder die sich in eine Ebene abwickeln lassen, beispielsweise Ebenen (azimutale Projektionen), Kegel (konische Projektionen) oder Zylinder (Zylinderprojektionen). Welche aus der Vielzahl von Projektionen geeignet ist, hängt v.a. von der Größe des Gebiets, dessen Lage auf der Erde (Polgebiete oder mittlere Breitengrade) sowie von der jeweiligen Aufgabenstellung ab.

Ziel ist es, eine Projektion zu finden, die möglichst kleine Verzerrungen verursacht. Dies kann dadurch erreicht werden, dass nur Teilstücke der Erdoberfläche abgebildet werden, oder man akzeptiert, dass nur teilweise Verzerrungsfreiheit vorliegt (z.B. nur 


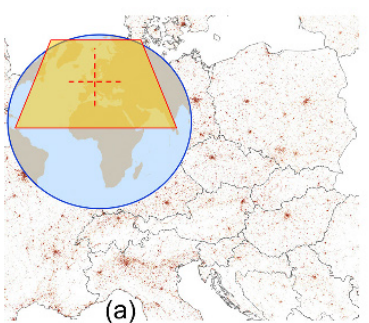

(a)

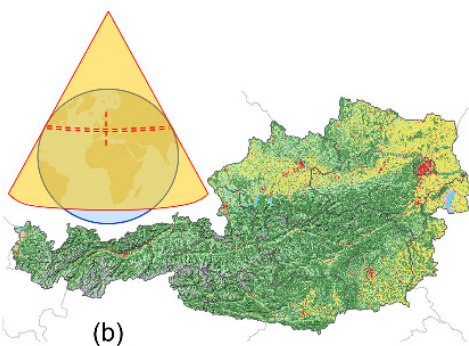

(b)

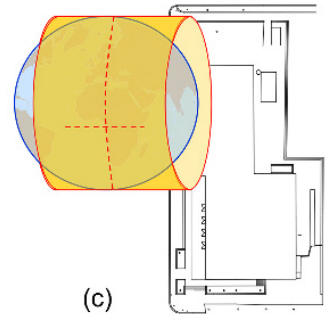

(c)

Abbildung 6.2.4: Anwendungsspezifische Projektionen: (a) flächentreue Azimutalprojektion für thematische Karten wie die Versiegelung in der EU (Versiegelungsdaten: EEA 2015), (b) winkeltreue Kegelprojektion (Lambert) für thematische Karten wie die Landbedeckung in Österreich (Karte der Landbedeckung: LISA 2017; Reliefschummerung: Jarvis et al. 2008), (c) winkeltreue transversale Zylinderprojektion (GaußKrüger) für die Erstellung detaillierter Pläne am Beispiel des Türkenwirtgebäudes auf dem BOKU-Gelände (Buchta und Zangl 2019)

Winkeltreue oder nur Flächentreue). Abbildung 6.2.4 zeigt Beispiele für Projektionen und deren Anwendungen.

Zusammenfassend lässt sich sagen, dass es bei der Verwendung von vorhandenen Geodaten oder bei der Erfassung von Geodaten nicht ausreicht, nur die Koordinaten zu kennen (Lage und Höhe). Vielmehr braucht man ausführliche Angaben darüber, welche Bezugsfläche verwendet wurde, wie diese an die Erde angepasst wurde und mit welcher Projektion die Objekte auf der Erdoberfläche in die Ebene abgebildet wurden.

\subsubsection{Arten von Geodaten}

Geodaten zeichnen sich durch die folgenden drei Aspekte aus, welche auch als „Säulen“ eines GIS bezeichnet werden:

- Geometrie: Die geometrische Information liefert den Raumbezug. Dieser ermöglicht es, Objekte in einem bekannten Koordinatensystem zu verorten. Aus der Geometrie können Informationen wie Flächeninhalte, Strecken und Winkel abgeleitet werden. Da die Daten reale oder abstrakte Objekte beschreiben, ist der Detaillierungsgrad, d.h. die Größe und Genauigkeit, mit denen ein Objekt im GIS abgebildet wird, ein wichtiger Aspekt bei den geometrischen Eigenschaften.

- Thematik: Thematische Informationen beschreiben die nichtgeometrischen Eigenschaften des Objekts. Sie lassen sich bei GIS in zwei Gruppen einteilen:

- Thematische Layer: Sie geben Auskunft darüber, welchem Thema ein GISObjekt (Punkt, Linie, Fläche) zugeordnet wird. Abhängig von der Fragestellung werden die Objekte in verschiedene Layer gegliedert und somit in unterschiedlichen Ebenen abgespeichert. 
- Attribute oder Sachdaten: Attribute verfeinern die thematische Information und erlauben es, jedem Objekt seine spezifischen Eigenschaften zuzuordnen.

- Topologie: Sie beschreibt die Beziehungen geometrischer Objekte zueinander, v.a. deren Nachbarschaftsbeziehungen.

Grundsätzlich werden Geodaten in Form von Vektor- oder Raster-GIS-Modellen abgespeichert. Ein Raster ist eine Matrixstruktur, bestehend aus Rasterelementen. Diese können quadratisch oder auch rechteckig sein und werden als Pixel (vom englischen Picture-Element) oder Grid-Elemente bezeichnet. In einer Rasterstruktur wird jedem Pixel ein Wert zugeordnet. Raster-GIS-Modelle eignen sich zum Abspeichern von räumlich kontinuierlichen Phänomenen (z.B. Lufttemperatur in ${ }^{\circ} \mathrm{C}$, Seehöhe in Metern, Humusgehalt des Bodens in \%).

Das Vektor-GIS-Modell ist anders aufgebaut: Hier ist der Punkt Träger der geometrischen Information und stellt (in der Regel über Koordinaten) den Raumbezug her, aus dem sich geometrische Aussagen wie Höhen, Entfernungen oder Flächeninhalte ableiten lassen. Eine Linie ist eine Verbindung von Punkten zu Vektoren. Den einzelnen Vektoren und Punkten kann auch eine Thematik zugeordnet sein. Eine Fläche wird durch eine in sich geschlossene Linie oder durch mehrere Einzelflächen abgegrenzt. Vektor-GIS-Modelle eignen sich für die Darstellungen von Objekten, die räumlich eindeutig zu verorten sind (z.B. Grenzen). Abbildung 6.2.5 veranschaulicht die Möglichkeit, Geodaten als thematische Layer abzubilden.

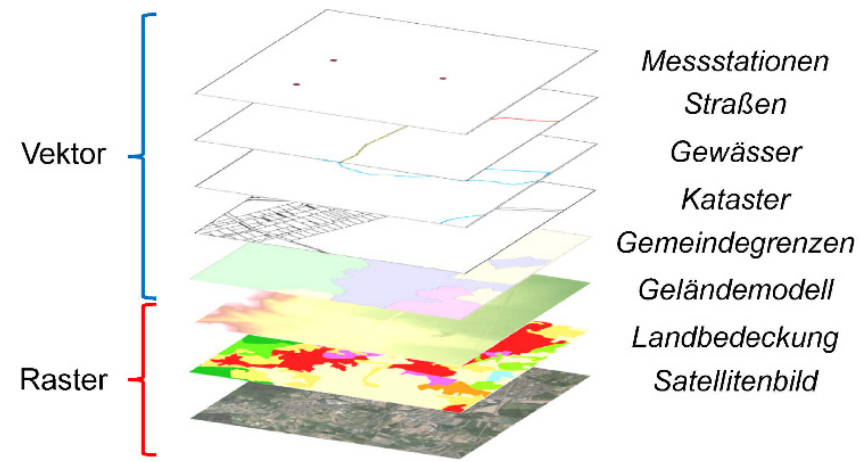

Abbildung 6.2.5: Prinzip eines GIS in Form von thematischen Layern mit unterschiedlichen Datentypen (Vektordaten als Punkt-, Linien- oder Flächenlayer bzw. Rasterdaten)

\subsubsection{Geoinformationssysteme}

GIS werden auch als raumbezogene Informationssysteme bezeichnet. Sie dienen der Erfassung, Speicherung, Verarbeitung und Darstellung aller Daten, die einen Teil der Erdoberfläche und die darauf befindlichen technischen und administrativen Einrich- 


\section{Umweltinformationssysteme \& -management}

tungen sowie ökologische und ökonomische Gegebenheiten beschreiben. Ein GIS umfasst Werkzeuge für die Verknüpfung und die Analyse der gespeicherten Daten, sodass Fragestellungen mit räumlichem Bezug beantwortet und Umweltprozesse simuliert werden können.

Die Anwendungsbereiche von GIS sind heute sehr breit gestreut. Gerade in den an der BOKU vertretenen Fachbereichen ist GIS heute ein nicht mehr wegzudenkendes Werkzeug geworden. Anwendungsbereiche finden sich z.B. in der Energie- und Wasserversorgung, bei Gebietskörperschaften, im Umweltbereich, in der Land- und Forstwirtschaft, im Geo-Marketing sowie im Risikomanagement.

Das folgende Beispiel (Abbildung 6.2.6) stammt aus einem EU-Projekt, dessen Ziel es war, den Alpen-Karpaten-Korridor für Wildtiere wieder durchgängig zu machen und somit eine ökologisch funktionsfähige Landschaft wiederherzustellen. Dabei wurde mithilfe von GIS-Modellierungen der Verlauf eines Korridors für Wildtiermigration berechnet. Die Ergebnisse dienen in weiterer Folge als Grundlage zur Erhaltung und Schaffung geeigneter Landschaftsstrukturen und Grünbrücken sowie für eine nachhaltige Raumplanung.
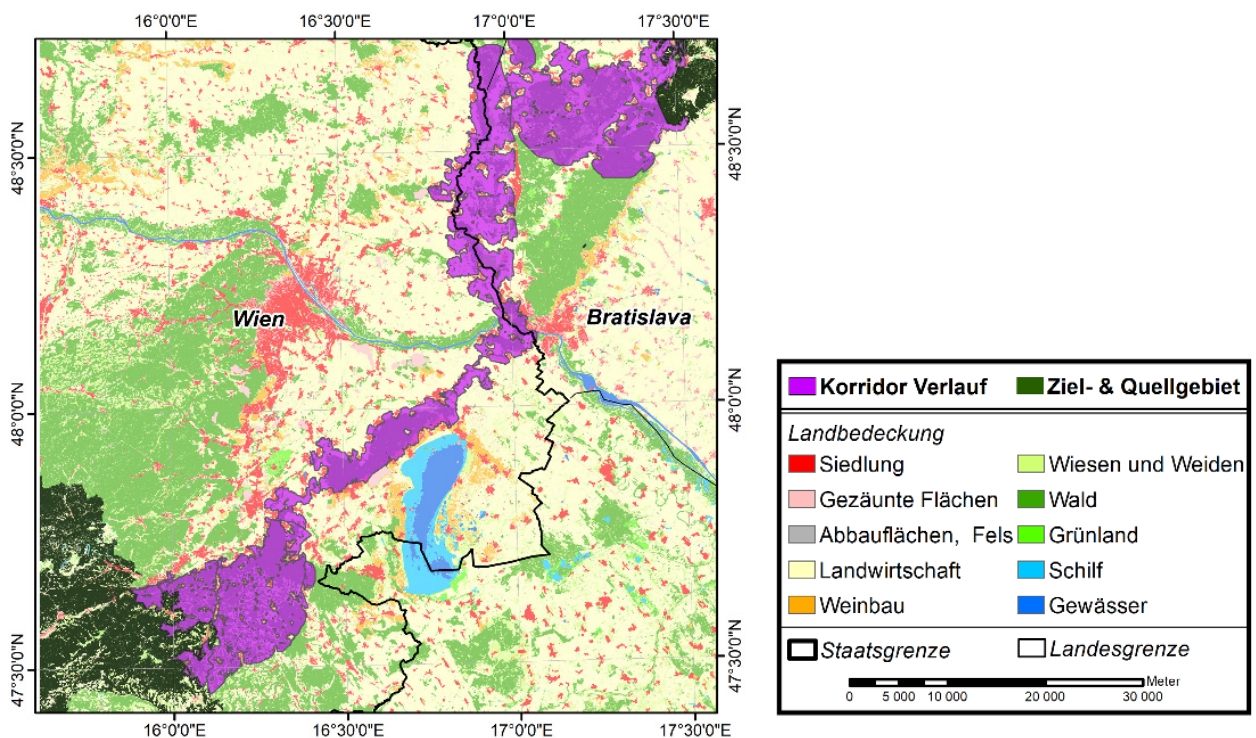

Abbildung 6.2.6: Beispiel für das Ergebnis einer GIS-Modellierung: Alpen-KarpatenKorridor für Wildtiere (modifiziert nach AKK 2014)

\subsubsection{Fernerkundung}

Die Fernerkundung beschäftigt sich mit der Beobachtung der Erde, ohne mit ihr in direkter Berührung zu stehen. Dazu werden Sensoren (Kameras und andere Messgeräte) 
an Satelliten, Flugzeugen oder Drohnen angebracht. Diese messen die von der Erdoberfläche reflektierte oder emittierte elektromagnetische Strahlung. Diese Information wird in Form von Bildern verfügbar gemacht.

Die meisten Fernerkundungssensoren nutzen das Sonnenlicht und erfassen jenen Anteil der Strahlung, welcher an der Erdoberfläche reflektiert wird. Die Auswertung solcher Bilder beruht darauf, dass spezifische Oberflächentypen (z.B. Vegetation, Wasser, Böden oder Gesteine) die Sonnenstrahlung in verschiedenen Wellenlängenbereichen unterschiedlich stark reflektieren.

Die besondere Stärke der Fernerkundung liegt in der systematischen flächendeckenden Erfassung von Phänomenen auf der Erde und ihrer Veränderung mit der Zeit. So dokumentieren Fernerkundungssensoren den Zustand der Erde schon seit den 1970er-Jahren. Abbildung 6.2.7 zeigt Beispiele für Bilder der US-amerikanischen Landsat-Satelliten.

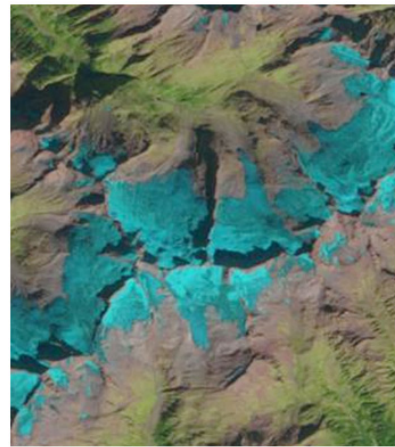

29.08.1985

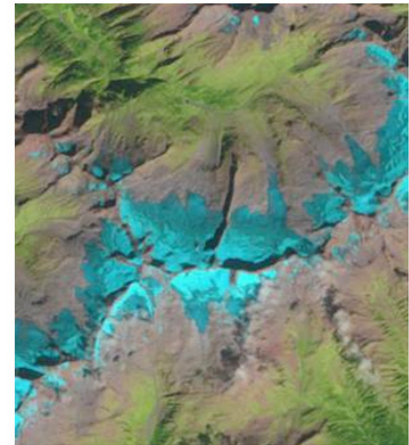

05.09.2005

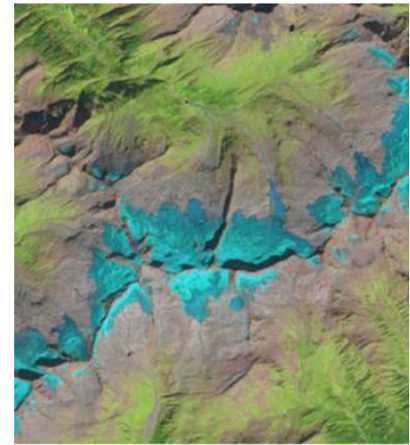

01.09.2015

Abbildung 6.2.7: Dokumentation des Rückgangs von Gletschern mit Landsat-Daten zu verschiedenen Zeitpunkten am Beispiel des Waxeggkees in den Zillertaler Alpen (USGS 2019)

\subsubsection{Spezifikation von Fernerkundungsdaten}

Die Eigenschaften von Fernerkundungssensoren und Fernerkundungsbilddaten lassen sich durch die verschiedenen Formen der „Auflösung" charakterisieren:

- Die räumliche Auflösung wird durch die Größe eines Bildelements (Pixels) am Boden charakterisiert. Die sogenannte „Ground Sample Distance“ gibt damit auch ungefähr die Größe der Details an, die man auf den Bildern noch erkennen kann.

- Die spektrale Auflösung gibt die Breite und die Anzahl der Spektralbereiche (Wellenlängenbereiche) an, in denen der Sensor die Strahlung erfasst. Sie beeinflusst die Unterscheidbarkeit und die Identifizierbarkeit verschiedener Oberflächen (Gesteine, Böden, Vegetation). 


\section{Umweltinformationssysteme \& -management}

- Die radiometrische Auflösung gibt die Genauigkeit an, mit der die Intensität der Strahlung innerhalb eines einzelnen Spektralbereichs gemessen werden kann - oder in anderen Worten: Sie wird durch die Anzahl der Intensitätsstufen definiert.

- Die zeitliche Auflösung gibt die Häufigkeit und den zeitlichen Abstand von Fernerkundungsaufnahmen desselben Gebiets an bzw. charakterisiert die Flexibilität in der Wahl der Aufnahmezeitpunkte.

\subsubsection{Methoden der Auswertung}

Zur Analyse von Fernerkundungsbildern müssen diese zunächst aufbereitet werden, um daraus die gewünschte Information ableiten zu können. So unterliegen die Bilddaten geometrischen Verzerrungen, welche beseitigt werden müssen. Diese lassen sich beispielsweise auf unebenes Gelände zurückführen oder sind sensorbedingt. Zudem müssen den Pixeln Koordinaten in einem der unter Abschnitt 6.2.2.1 angesprochenen Koordinatensysteme zugeordnet werden.

Die am Sensor ankommende Strahlung unterliegt zahlreichen Störeinflüssen. Die Störungen resultieren daraus, dass die Strahlung die Atmosphäre zweimal passieren muss, bevor sie vom Sensor aufgezeichnet wird: (i) von der Sonne durch die Erdatmosphäre bis zum Auftreffen auf die Erde und (ii) auf dem Weg zurück zum Weltall. Dies bringt eine Veränderung der am Sensor erfassten Strahlung mit sich, die nicht auf die Oberflächeneigenschaften zurückzuführen ist. Solche Störungen müssen unbedingt korrigiert werden, um die Reflexionseigenschaften der Oberfläche bestimmen zu können.

Nach diesen Schritten kann die eigentliche Auswertung der fernerkundlichen Bilddaten erfolgen. Oft besteht die Aufgabenstellung darin, eine thematische Karte zu erstellen. Dabei wird jedem Bildelement im Fernerkundungsbild eine thematische Kategorie zugewiesen. Ein Beispiel hierfür ist eine Landbedeckungskarte mit den thematischen Kategorien Gewässer, Wald, Wiese und versiegelte Flächen. Dabei können zwei Herangehensweisen unterschieden werden: die visuelle Bildinterpretation und automatisierte Auswerteverfahren.

Die visuelle Bildinterpretation umfasst Methoden, die nichtgeometrische Eigenschaften von Objekten ermitteln und den Bildinhalt deuten. Dafür werden v.a. räumlich hochauflösende Luft- oder Satellitenbilder herangezogen. Dabei befassen sich Interpretinnen und Interpreten im Wesentlichen mit folgenden Teilaufgabestellungen:

(1) Identifizieren von Objekten, die ein ähnliches, möglichst homogenes Erscheinungsbild in den Bilddaten haben,

(2) Feststellen von Objekteigenschaften,

(3) Erkennen von funktionalen Zusammenhängen. 
Für die Aufgaben (1) und (2) werden üblicherweise Objektmerkmale wie Größe, Gestalt oder Form, aber auch Farbgebung und Feinstruktur herangezogen. Hilfreich ist dabei auch ein eventuell vorhandener Schattenwurf. Er erleichtert die Abschätzung von Höhen aus dem Schlagschatten einzeln stehender Objekte wie Bäume. Die Deutung von räumlichen Mustern oder Nachbarschaftsbeziehungen (z.B. Siedlungsmuster, Muster landwirtschaftlicher Kulturen) erfordert detaillierte Kenntnisse in der jeweiligen Fachrichtung.

Automatisierte Auswerteverfahren zielen hingegen darauf ab, relevante Informationen automatisiert abzuleiten. Bei der digitalen Klassifikation werden den Objekten im Bild anhand ihrer Eigenschaften Kategorien zugeordnet, ähnlich wie bei der Bildinterpretation. Allerdings erfolgt die Zuweisung mithilfe von Algorithmen, die in Softwarepaketen umgesetzt oder selbst programmiert wurden.

Neben der Erstellung von Landbedeckungskarten beschäftigt sich die Fernerkundung auch mit der Untersuchung von Veränderungen auf der Erdoberfläche. Durch den Vergleich von Landbedeckungskarten zu verschiedenen Zeitpunkten kann beispielsweise das Schmelzen von Gletschern oder Eismassen dokumentiert werden (siehe Beispiel Zillertaler Alpen in Abbildung 6.2.7). Fernerkundungsmethoden erlauben es auch, physikalische Parameter und deren Veränderung zu erfassen. So kann der Anstieg von Temperaturen oder die Erwärmung der Meere quantifiziert werden.

Die Untersuchung von Zeitreihen, also die Beobachtung einer Größe in regelmäßigen Abständen (z.B. wöchentlich) über mehrere Jahre, erlaubt die Erfassung von Veränderungen zwischen einzelnen Jahren und auch die Ableitung von langfristigen Trends.

Ein Fallbeispiel ist die Beobachtung des Eintrittszeitpunkts des Blattwachstums im Frühjahr. Dies wird in Abbildung 6.2.8 am Beispiel von Österreich dargestellt. Traditionell wird der Beginn des Frühjahrs durch sogenannte Zeigerpflanzen (z.B. Blüte der Hasel) von freiwilligen Beobachterinnen und Beobachtern erfasst (vgl. ZAMG 2013). Solche Beobachtungen sind sehr aufwendig und werden nur an einzelnen Standorten für bestimmte Wildpflanzen oder landwirtschaftliche Kulturpflanzen durchgeführt. Die Fernerkundung gestattet eine flächendeckende Abschätzung solcher Zeitpunkte und veranschaulicht somit die räumliche Verteilung dieser Größen. In Abbildung 6.2.8 wird gezeigt, dass der Eintritt des Blattwachstums sich zwischen 2002 und 2017 tendenziell in Richtung eines früheren Zeitpunkts verschoben hat. Dies ist ein signifikanter Hinweis auf den Klimawandel.

Der Beitrag der Fernerkundung zur Umsetzung der nachhaltigen Entwicklungsziele der Agenda 2030 (SDGs) ist darin zu sehen, dass sie den derzeitigen Zustand und 


\section{Umweltinformationssysteme \& -management}

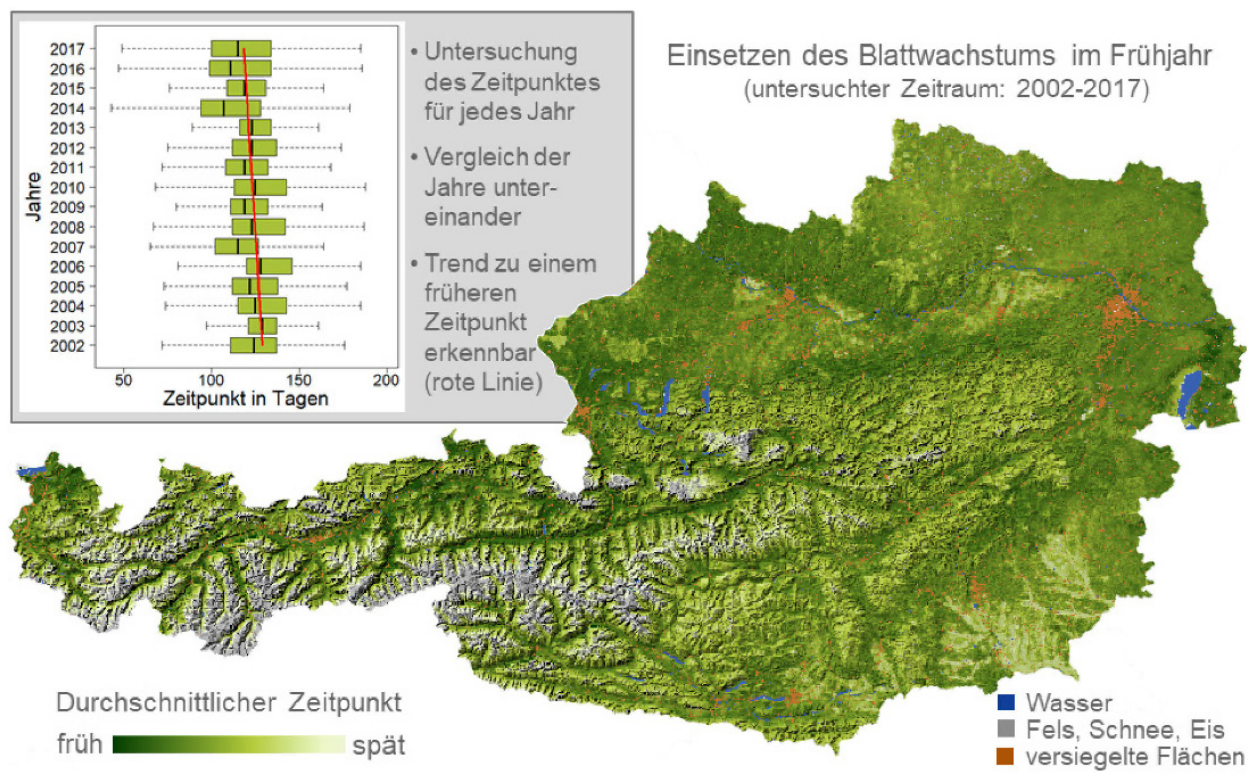

Die Aufnahme des Vegetationsverlaufs in Satellitenbildern alle 8 Tage erlaubt die Bestimmung des Eintrittszeitpunkts für jeden Bildpunkt (ca. $1 \mathrm{~km}$ ) in jedem Jahr.

Abbildung 6.2.8: Untersuchung des Einsetzens des Blattwachstums im Frühjahr mithilfe von Zeitreihen der MODIS-Sensoren (Moderate-resolution Imaging Spectroradiometer) (MODIS-Daten: LP DAAC 2019; Landbedeckung: LISA 2017; Reliefschummerung: Jarvis et al. 2008)

die Auswirkungen von ergriffenen Maßnahmen mithilfe von Indikatoren dokumentiert und überwacht. Für einige dieser Indikatoren werden die erforderlichen Daten schon routinemäßig bereitgestellt. Ein Beispiel hierfür ist die Erfassung von Waldgebieten für das SDG 15 (Landökosysteme schützen, wiederherstellen und ihre nachhaltige Nutzung fördern; vgl. Anderson et al. 2017; ESA 2018b, S. 13ff.). Für andere Indikatoren ist die Methodik zwar vorhanden, die Daten werden aber noch nicht regelmäßig abgeleitet. Für weitere Indikatoren ist die entsprechende Methodik noch Forschungsgegenstand, hier leitet sich ein hoher Bedarf an Weiterentwicklungen ab. Vor diesem Hintergrund spannt sich ein interessantes und breites Tätigkeitsfeld für UBRM-Absolventinnen und -Absolventen auf.

Zusammenfassend lässt sich sagen, dass Fernerkundung und GIS wichtige Werkzeuge zur kontinuierlichen und verlässlichen Messung und Dokumentation von Phänomenen auf der Erde sowie von Umwelteinflüssen und deren Konsequenzen sind. Sie erlauben damit die Erfassung mittel- und langfristiger Trends und ein Monitoring von Veränderungen. 


\section{Literatur}

AKK (Alpen Karpaten Korridor) (2014): Wildökologische GIS-Modellierung des Korridorverlaufes im Rahmen des Projektes Alpen Karpaten Korridor, gefördert durch die EU im Rahmen des ETZ Slowakei-Österreich, das Land Niederösterreich, das Land Burgenland und das Lebensministerium. Verfügbar in: http://www.alpenkarpatenkorridor.at/ [Abfrage am 20.7.2019].

Anderson, K., Ryan, B., Sonntag, W., Kavvada, A., and Friedl, L. (2017): Earth observation in service of the 2030 Agenda for Sustainable Development. Geo-spatial Information Science, 20, 2, 77-96. https://doi.org/10.1080/10095020.2017.1333230.

Buchta, B. und Zangl, C. (2019): Geodätische Bestandsaufnahme und Kartierung des Bereichs TÜWI. Konstruktives Projekt, Universität für Bodenkultur Wien, unveröffentlicht.

EEA (European Environment Agency) (2015): High Resolution Layer: Imperviousness Density (IMD) 2015. Available at: https://land.copernicus.eu/pan-european/high-resolutionlayers/imperviousness/status-maps/2015 [accessed 23.8.2019].

ESA (European Space Agency) (2018a): Earth's geoid as seen by GOCE. Available at: https://www.esa.int/spaceinimages/Images/2008/05/Earth s geoid as seen by GOCE2 [accessed 23.8.2019].

ESA (European Space Agency) (2018b): Satellite Earth Observations in Support of the Sustainable Development Goals. The CEOS Earth Observation Handbook, Special 2018 Edition. Available at: http://eohandbook.com/sdg/ [accessed 18.3.2019].

GADM (Database of Global Administrative Areas) (2018): Global administrative data version 3.6. Available at: https://gadm.org/ [accessed 23.8.2019].

Jarvis A., Reuter, H. I., Nelson, A., and Guevara, E. (2008): Hole-filled seamless SRTM data V4. International Centre for Tropical Agriculture (CIAT). Available at: http://srtm.csi.cgiar.org [accessed 23.8.2019].

LISA (Land Information System Austria) (2017): Landbedeckungskarte Österreich. Verfügbar in: https://www.landinformationsystem.at/ [Abfrage am 20.7.2019].

LP DAAC (Land Processes Distributed Active Archive Center) (2019): MODIS data distributed by the LP DAAC located at USGS/EROS, Sioux Falls, SD, USA. Available at: https://pdaac.usgs.gov/ [accessed 23.8.2019].

Stadt Wien und Österreichische Länder bzw. Ämter der Landesregierung (2016): basemap.at - die Verwaltungsgrundkarte von Österreich. Verfügbar in: https://basemap.at/ [Abfrage am 20.7.2019].

USGS (U.S. Geological Survey) (2019): Landsat data available from the USGS. Reston, VA, USA. Available at: https://earthexplorer.usgs.gov/ [accessed 23.8.2019].

ZAMG (Zentralanstalt für Meteorologie und Geodynamik) (Hrsg.) (2013): Beobachtungsanleitung für die Phänologie. Wien. Verfügbar in:

http://www.phenowatch.at/fileadmin/ migrated/content uploads/Beobachtungsanleitung 2015 8.pdf [Abfrage am 18.3.2019].

\section{Weiterführende Literatur}

Albertz, J. (2019): Einführung in die Fernerkundung: Grundlagen der Interpretation von Luft- und Satellitenbildern. 5. akt. Auflage, Darmstadt: WBG (Wissenschaftliche Buchgesellschaft).

Bill, R. (2016): Grundlagen der Geo-Informationssysteme. 6., völlig neu bearb. u. erw. Auflage. Karlsruhe: Wichmann. 
Open Access Dieses Kapitel wird unter der Creative Commons Namensnennung - Nicht kommerziell 4.0 International Lizenz (http://creativecommons.org/licenses/by-nc/4.0/deed.de)veröffentlicht, welche die nicht-kommerzielle Nutzung, Vervielfältigung, Bearbeitung, Verbreitung und Wiedergabe in jeglichem Medium und Format erlaubt, sofern Sie den/die ursprünglichen Autor(en) und die Quelle ordnungsgemäß nennen, einen Link zur Creative Commons Lizenz beifügen und angeben, ob Änderungen vorgenommen wurden.

Die in diesem Kapitel enthaltenen Bilder und sonstiges Drittmaterial unterliegen ebenfalls der genannten Creative Commons Lizenz, sofern sich aus der Abbildungslegende nichts anderes ergibt. Sofern das betreffende Material nicht unter der genannten Creative Commons Lizenz steht und die betreffende Handlung nicht nach gesetzlichen Vorschriften erlaubt ist, ist auch für die oben aufgeführten nicht-kommerziellen Weiterverwendungen des Materials die Einwilligung des jeweiligen Rechteinhabers einzuholen.

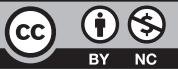

